\title{
Low-level laser therapy with helium- neon laser improved viability of osteoporotic bone marrow-derived mesenchymal stem cells from ovariectomy-induced osteoporotic rats
}

Somaye Fallahnezhad

Abbas Piryaei

Faraj Tabeie

Hamid Nazarian

Hasan Darbandi

Abdoldllah Amini

Ataroalsadat Mostafavinia

Seyed Kamran Ghorishi

Ali Jalalifirouzkouhi

Mohammad Bayat 


\title{
Low-level laser therapy with helium-neon laser improved viability of osteoporotic bone marrow-derived mesenchymal stem cells from ovariectomy-induced osteoporotic rats
}

\author{
Somaye Fallahnezhad, ${ }^{a}$ Abbas Piryaei, ${ }^{a}$ Faraj Tabeie, ${ }^{b}$ Hamid Nazarian, ${ }^{a}$ Hasan Darbandi, ${ }^{c}$ Abdoldllah Amini, ${ }^{a}$ \\ Ataroalsadat Mostafavinia, ${ }^{a}$ Seyed Kamran Ghorishi, ${ }^{d}$ Ali Jalalifirouzkouhi, ${ }^{e}$ and Mohammad Bayat ${ }^{a, *}$ \\ a Shahid Beheshti University of Medical Sciences, School of Medicine, Department of Biology and Anatomical Sciences, Koodakyar Street, \\ Danshjoo Boulevard, Velenjak, Shahid Chamran Highway, PO Box 19395/4719, Tehran 1985717443, Iran \\ ${ }^{b}$ Shahid Beheshti University of Medical Sciences, Physiotherapy Research Centre, School of Rehabilitation Sciences, Department of \\ Basic Sciences, and School of Medicine, Department of Nuclear Medicine, Damavand Street across from Bu Ali Hospital, Tehran, Iran \\ 'Shahid Beheshti University of Medical Sciences, School of Medicine, Department of Immunology, Koodakyar Street, Danshjoo Boulevard, \\ Velenjak, Shahid Chamran Highway, PO Box 19395/4719, Tehran 1985717443, Iran \\ dQom University, Department of Statistics, Faculty of Sciences, Old Road of Isfahan, Qom 3716146611, Iran \\ eShahid Beheshti University of Medical Sciences, Cellular and Molecular Biology Research Center, School of Medicine, Koodakyar Street, \\ Danshjoo Boulevard, Velenjak, Shahid Chamran Highway, PO Box 19395/4719, Tehran 1985717443, Iran
}

\begin{abstract}
The purpose of this study was to evaluate the influences of helium-neon ( $\mathrm{He}-\mathrm{Ne})$ and infrared (IR) lasers on the viability and proliferation rate of healthy and ovariectomy-induced osteoporotic (OVX) bone marrow mesenchymal stem cells (BMMSCs) in vitro. MSCs harvested from the BM of healthy and OVX rats were culture expanded. He-Ne and IR lasers were applied three times at energy densities of $0.6,1.2$, and $2.4 \mathrm{~J} / \mathrm{cm}^{2}$ for BMMSCs. BMMSCs viability and proliferation rate were evaluated by MTT assay on days 2, 4, 6, 14, and 21. The results showed that healthy BMMSCs responded optimally to $0.6 \mathrm{~J} / \mathrm{cm}^{2}$ using an IR laser after three times of laser radiation. Moreover, it was found that OVX-BMMSCs responded optimally to $0.6 \mathrm{~J} / \mathrm{cm}^{2}$ with $\mathrm{He}-\mathrm{Ne}$ laser and one-time laser radiation. It is concluded that the low-level laser therapy (LLLT) effect depends on the physiological state of the BMMSCs, type of the laser, wavelength, and number of laser sessions. The biostimulation efficiency of LLLT also depends on the delivered energy density. LLLT can enhance the viability and proliferation rate of healthy and especially osteoporotic autologous BMMSCs, which could be very useful in regenerative medicine. ๑ 2016 Society of Photo-Optical Instrumentation Engineers (SPIE) [DOI: 10.1117/1.JBO.21.9.098002]
\end{abstract}

Keywords: mesenchymal stem cells; low-level laser therapy; osteoporosis; ovariectomy; viability; proliferation rate; rat. Paper 160361R received Jun. 15, 2016; accepted for publication Sep. 13, 2016; published online Sep. $29,2016$.

\section{Introduction}

The regenerative bone formation arm ultimately declines in postmenopausal osteoporosis (OP). ${ }^{1}$ OP is shown by low bone mineral density (BMD) and abnormal bone architecture. Common fracture sites are vertebras, femural neck (hip), and distal parts of ulna and radius. Unfortunately, OP is underdiagnosed and undertreated. ${ }^{2} \mathrm{OP}$ and its consequence of low trauma fractures (pathologic fractures) produce a major health burden in aging populations, because they lead to increase morbidity, mortality, ${ }^{3}$ and health care costs. The most costly fracture is arguably a hip fracture. ${ }^{4}$ Ten million Americans are currently affected by OP which is responsible for more than 1.5 million fractures annually. The direct medical cost of OP is estimated to be 17 to 20 billion dollars annually. Indeed, it has been predicted that the total number of hip fractures worldwide will increase from 1.26 million in 1990 to 2.6 million by 2025 and to 4.5 million by $2050 .^{5}$ The current therapeutic protocols do not adequately address this problem. ${ }^{1}$

In OP, increased susceptibility to fractures results from insufficient osteoblastogenesis and increased osteoclastogenesis. ${ }^{5}$

*Address all correspondence to: Mohammad Bayat, E-mail: mohbayat@sbmu. ac.ir
Prall et al. $^{6}$ reported that osteoporotic mesenchymal stem cell (MSC) featured an accurate phosphorylation pattern of SMAD1/5/8, but a significantly reduced activation of extracellular signal-regulated kinases (ERK)1/2 by bone morphogenetic protein (BMP-2) stimulation. Furthermore, osteoporotic MSC showed significantly reduced basal expression levels of Runtrelated transcription factor (Runx-2) and distal-less homeobox 5 gene (Dlx5). Moreover, the global osteogenic differentiation in standard osteogenic differentiation media was reduced in osteoporotic MSC. Nevertheless, osteoporotic MSC was shown to feature an adequate induction of osteogenic differentiation due to BMP-2 stimulation. It was shown that BMP-2 and BMP-7 may represent an effective therapeutic agent for the treatment of fractures in osteoporotic patients. ${ }^{6,7}$ In ovariectomy-induced osteoporosis (OVX) in rats, OVX was associated with reduced bone marrow MSC (BMMSC) number and its growth, and MSC shows impaired engraftment to fracture and osteoinduction in addition to disordered bone regeneration. ${ }^{8}$

Low-level laser therapy (LLLT) has been used in many experiments to stimulate cell proliferation. Low-level red light (laser) ranges from 630 to $690 \mathrm{~nm}$ [e.g., helium-neon $(\mathrm{He}-\mathrm{Ne})$

$1083-3668 / 2016 / \$ 25.00$ @) 2016 SPIE 
lasers] and infrared (IR) light ranges from $800 \mathrm{~nm}$ or above (800 to $1400 \mathrm{~nm}$; e.g., IR laser). Cells such as fibroblasts, lymphocytes, keratinocytes, and osteoblasts have indicated growth proliferation when subjected to laser radiation. But little is known about the effects of LLLT on SC. Thus, by a systematic literature review, the effects of LLLT on the in vitro proliferation of MSCs were assessed. In most studies, the biostimulatory effect of LLLT on the proliferation rate of the irradiated cells was demonstrated. ${ }^{9}$ LLLT has a photochemical effect that is comparable to photosynthesis in plants according to the light absorbed and exerts a chemical change. ${ }^{9}$ The cellular and molecular mechanisms of LLLT suggest that the photons are absorbed in the mitochondria by cytochrome-c oxidase ${ }^{10}$ and they are in charge of the cellular response to IR and red visible light. LLLT stimulates adenosine triphosphate (ATP) production and low levels of reactive oxygen species (ROS) (free radicals), activates transcription factors such as nuclear factor kappa beta $(\mathrm{NF}-\kappa \mathrm{B})$, and induces transcription of many products responsible for the beneficial effects of LLLT. ${ }^{11}$

The escalation in ATP is a primary effect that leads to a cascade of events including cellular proliferation and cytoprotection. ${ }^{12} \mathrm{He}-\mathrm{Ne}$ laser is applied in clinical practices and experimental studies to accelerate skin wound healing in healthy and diabetic subjects. ${ }^{13}$ The development of IR lasers, delivering about $830 \mathrm{~nm}$, by providing a better tissue penetration than $\mathrm{He}-\mathrm{Ne}$ visible laser, offers new putative targets to LLLT such as bone healing. ${ }^{14} \mathrm{~A}$ recent in vivo report has described the positive effects of IR $(830 \mathrm{~nm})$ laser in the treatment of femurs of osteopenic rats. ${ }^{15}$ It was reported that in vitro osteoblast proliferation increased significantly after IR laser radiation. ${ }^{16}$

MSCs have the ability to generate cells of connective tissue lineages including fat, bone, cartilage, and muscle. These cells have received a great deal of interest due to their potential use in tissue engineering and regenerative medicine. ${ }^{17}$ LLLT has been shown to stimulate the growth, proliferation, and differentiation of SCs in vitro. ${ }^{18-20} \mathrm{He}-\mathrm{Ne}$ laser radiation with $2 \mathrm{~J} / \mathrm{cm}^{2}$ energy density improved the proliferation and migration of human epidermal SC in vitro. ${ }^{18}$ While some studies have reported the beneficial effects of LLLs using IR laser $(810 \mathrm{~nm})$ with 1 to $4 \mathrm{~J} / \mathrm{cm}^{2}$ energy density for improvement of the proliferation of MSC and BMMSCs in vitro, ${ }^{19}$ and stimulation of BMMSCs differentiation into osteoblasts, ${ }^{20}$ other work has reported that using an IR laser with $808 \mathrm{~nm}$ and $4 \mathrm{~J} / \mathrm{cm}^{2}$ did not alter mice bone progenitor cell proliferation and differentiation. ${ }^{21}$

To the best of the authors' knowledge, no study has investigated the effects of LLLT on OVX-BMMSC. The aim of this study was to assess the effects of two types of LLLs using $\mathrm{He}-\mathrm{Ne}$ and IR lasers on viability and proliferation rate of OVXBMMSC in vitro. It is important to note that it was recently reported that transplanting in vitro cultured BMMSCs with appropriate osteogenic phenotype into sites, particularly those at risk of developing osteoporotic bone, improved bone structure and enhanced its biomechanical properties. ${ }^{22}$ On the other hand, allogeneic MSCs are indicated as cells that are responsible for exerting wide ranging and local signals predominantly suppress and affect innate and adaptive immunity. Nonetheless, these cells also keep a degree of immunogenicity in some conditions, which may limit MSC longevity and reduce their beneficial effects. ${ }^{23}$ Thus, laser-treated osteoporotic autologous BMMSCs may represent a promising therapeutic agent for the treatment of $\mathrm{OP}$ and fractures in osteoporotic patients.

\section{Materials and Methods}

\subsection{Animals and Surgery}

Twelve 14-week-old female Wistar rats weighing about $200 \mathrm{~g}$ were obtained from Animal House at the Iranian branch in the Pasteur Institute, Tehran, Iran. The rats were fed a standard diet and water ad libitum, and were held in a cage located in a controlled animal room environment for the duration of the experiment. They were randomized into two groups to undergo either a sham surgery (sham group) or bilateral ovariectomization (OVX group). All the procedures were approved by the Medical Ethics Committee of Shahid Beheshti University of Medical Sciences, Tehran, Iran (protocol no. 1393-1-91-13223).

\subsection{Ovariectomy}

The OVX rats were subjected to total ovariectomy. ${ }^{24}$ OVX was carried out by two paravertebral skin incisions while the rats were under general anesthesia. The rats were anesthetized by intramuscular injections of $50 \mathrm{mg} / \mathrm{kg}$ ketamine hydrochloride (Rotex Medica, Tritteu, Germany) together with $5 \mathrm{mg} / \mathrm{kg} \mathrm{diaz-}$ epam (Caspian, Rasht, Iran). The uterine tubes were ligated, and following the removal of the ovaries, the incisions were closed (Fig. 1). For antibiotic therapy, Ceftriaxone (Jaber ben Hayan, Tehran, Iran) at a dose of $50 \mathrm{mg} / \mathrm{kg}$ was injected immediately before and at 24 and $48 \mathrm{~h}$ after surgery. At 3.5 months postsurgery, ${ }^{25}$ the rats of both groups were submitted for computed tomography (CT) scanning examination in order to establish OP.

\subsection{CT Scanning}

OP development of OVX rats was evaluated qualitatively by CT scanning using multislides $[\mathrm{kv}=100$, ma $=50$, sections $=$ $2 \mathrm{~mm}$, field of view $=240 \mathrm{~mm}$, Toshiba, Aquilion 16, Japan] as compared to that of the sham rats. Tibial and femoral bones' density of rats was determined under general anesthesia. All the bones were observed by an expert radiologist, and bone densities were reported in Hounsfield units (HU). ${ }^{26}$ The radiologist was unaware of the group division.

\subsection{BMMSCs Preparation and Cell Culture}

All the rats were euthanized and BMMSCs were harvested from the bilateral tibias and femurs. Briefly, rats were euthanized with an overdose of the anesthesia, then the bones were excised aseptically, and the BM flushed from the shaft. After centrifugation for $4 \mathrm{~min}$ at $1200 \mathrm{rpm}$ and $4^{\circ} \mathrm{C}$, the supernatants were discarded and the cell pellets were resuspended in complete medium supplemented with $10 \%$ fetal bovine serum and $1 \%$ penicillin/ streptomycin (all obtained from Biovest). The cultures were incubated at $37^{\circ} \mathrm{C}$ with $5 \% \mathrm{CO}_{2}$ in a humidified atmosphere and nonadherent cells were removed after $48 \mathrm{~h}$. When the cells reached $80 \%$ to $90 \%$ confluence, they were harvested and diluted at $1: 2$ for further expansion. Cells at passage- 4 were used in the experiments.

\subsection{Characterization of BM-Derived Adherent Cells by Immunophenotyping}

To detect surface antigen (Ag), passage-4 aliquots of adherent cells $\left(1 \times 10^{5}\right.$ cells $\left./ \mathrm{ml}\right)$ were washed twice with phosphatebuffered saline (PBS) after detachment with $0.25 \%$ trypsinEDTA. The cell pellets were diluted with PBS and incubated 


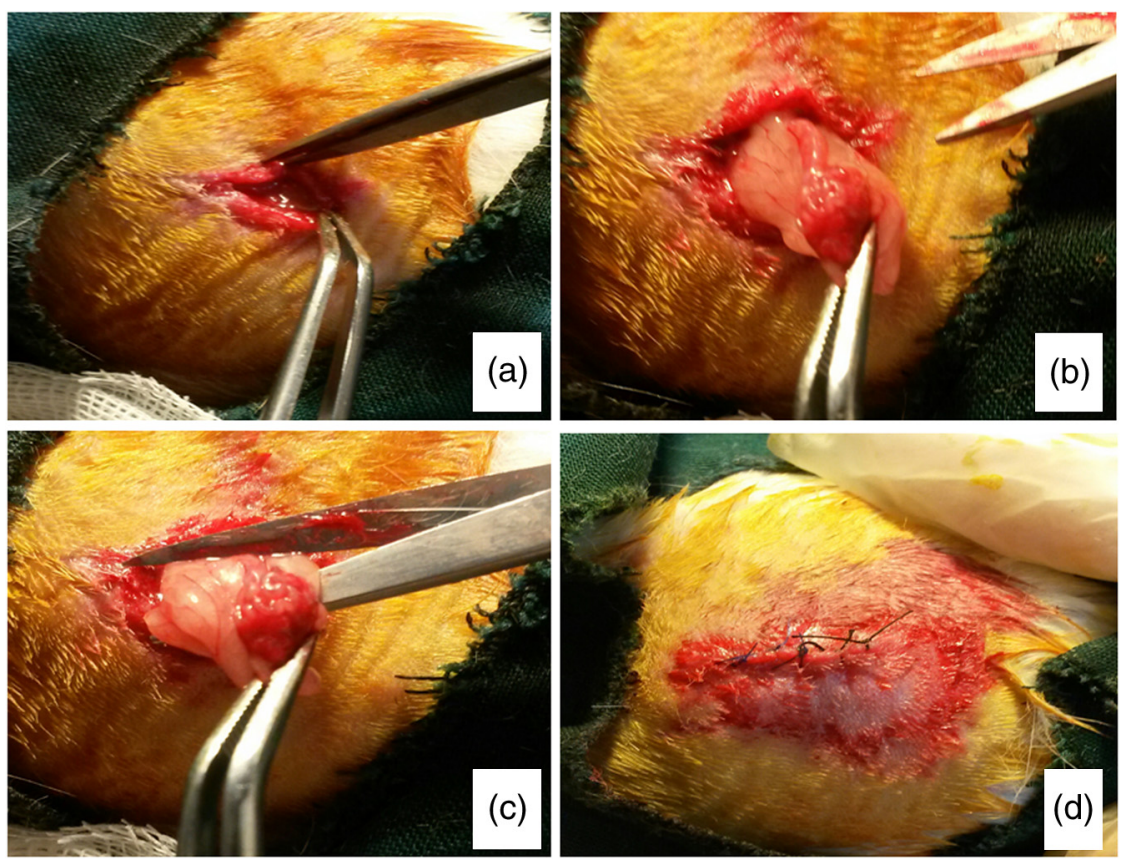

Fig. 1 Stages of ovariectomy. The images show (a) the incision, (b) exposed ovary, (c) removed ovary and sutured abdominal end of the ovarian tube, and (d) sutured abdominal wall and skin.

with fluorescein isothiocyanate-conjugated antibody $(\mathrm{Ab})$ for 30 min (Becton Dickinson, California). The antibodies (Ab) were used for positive staining for surface markers of MSCs (CD73, CD90, and CD105) and negative staining for markers of hematopoietic SCs (CD34 and CD45). As an isotype control, PE-conjugated nonspecific mouse IgG was used. After incubation, before being rarefied again with a sheath fluid, the researchers washed the cells again with PBS and subjected them to flow cytometric analysis.

\subsection{Low-Level Laser Therapy}

The culture medium was replaced by fresh medium immediately before radiation. The whole well was exposed to laser. The continuous wave lasers used were $\mathrm{He}-\mathrm{Ne}$ laser (IR-2000; Iranian Atomic Energy Agency, Tehran, Iran) and IR laser (NI150p, Noura instruments, Tehran, Iran). The He-Ne laser beam was expanded by a biconvex lens and collimated by another biconvex lens. ${ }^{27}$ The spot size of the IR laser was adjusted by a coneshaped pine hole collimator to cover a 15.6-mm well. In all the experiments, the He-Ne laser source and the IR laser were fixed vertically with a holder at a distance of 15 and $10 \mathrm{~cm}$, respectively, above the culture medium, which made the diameter of the laser $15.6 \mathrm{~mm}$ (corresponding to the 15.6-mm diameter of culture dish) (Fig. 2). The outputs of the $\mathrm{He}-\mathrm{Ne}$ laser and IR laser were controlled by a calibrated power meter made by the Iranian Atomic Energy Agency and another power meter made by OPHIR, 10A-V2-SH, respectively. The powers of the lasers at the surface of the culture medium were measured. The specifications of the laser groups are shown in Table 1. The cells were plated in such a way that one well, between the seeded wells, was left empty to avoid the accidental dispersion of light between wells during laser radiation. The cover of the plate was removed during the radiation period and black backgrounds of
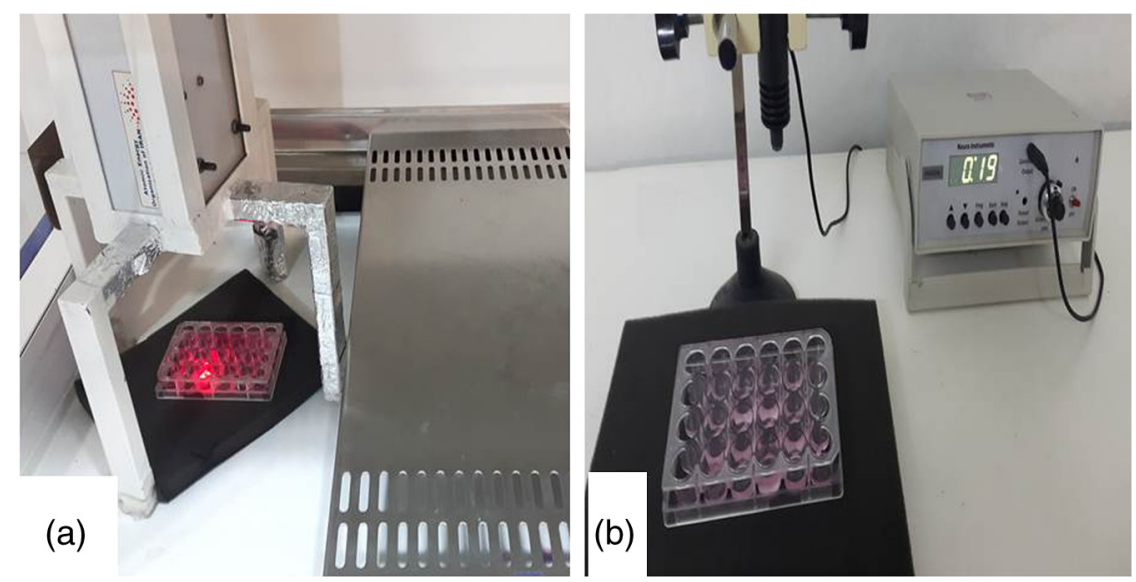

Fig. 2 (a) A He-Ne and (b) IR lasers, which were prepared for the study. 
Table 1 The specifications of the lasers used.

\begin{tabular}{|c|c|c|c|c|c|c|c|c|c|c|c|}
\hline \multirow{2}{*}{$\frac{\text { Laser type }}{\mathrm{He}-\mathrm{Ne}}$} & \multirow{2}{*}{$\begin{array}{c}\begin{array}{c}\text { Wavelength } \\
(\mathrm{nm})\end{array} \\
632.8\end{array}$} & \multirow{2}{*}{$\begin{array}{c}\begin{array}{c}\text { Power } \\
(\mathrm{mW})\end{array} \\
3\end{array}$} & \multicolumn{3}{|c|}{$\begin{array}{c}\text { Energy densities } \\
\left(\mathrm{J} / \mathrm{cm}^{2}\right)\end{array}$} & \multicolumn{3}{|c|}{ Times (s) } & \multirow{2}{*}{$\begin{array}{c}\begin{array}{c}\text { Laser beam } \\
\text { diameter }(\mathrm{cm})\end{array} \\
1.56\end{array}$} & \multirow{2}{*}{$\begin{array}{c}\begin{array}{c}\text { Laser beam } \\
\text { surface area }\left(\mathrm{cm}^{2}\right)\end{array} \\
1.91\end{array}$} & \multirow{2}{*}{$\begin{array}{c}\begin{array}{c}\text { Power density } \\
\left(\mathrm{W} / \mathrm{cm}^{2}\right)\end{array} \\
0.0016\end{array}$} \\
\hline & & & 0.6 & 1.2 & 2.4 & 378 & 756 & 1512 & & & \\
\hline IR & 810 & 50 & 0.6 & 1.2 & 2.4 & 23 & 45 & 84 & 1.56 & 1.91 & 0.0261 \\
\hline
\end{tabular}

irradiated areas were used to minimize light reflections in all the experiments. Control cells underwent the same procedure as the irradiated groups except that the laser was not turned on. Each control and laser-treated group consisted of three wells. Laser radiation was performed thrice every other day inside a clean class two laminar flow hood. Cells were harvested at $2,4,6,14$, and 21 days post first radiation. The cells were maintained in $\mathrm{CO}_{2}$ incubator during the experiment without any treatment thereafter.

\subsection{Cell Viability}

At 2, 4, 6, 14, and 21 days after laser radiation, surviving cells were counted by the MTT assay [3-(4,5-dimethylthiazol-2-yl)2,5-diphenyltetrazolium bromide]. This assay detected surviving cultured cells and the degree to which they were activated. There were about $3 \times 10^{3}$ cells per well. At each time point, for the MTT assay, the culture medium was removed and $450 \mu \mathrm{l}$ of fresh culture medium and $50 \mu \mathrm{l}$ of MTT $(5 \mathrm{mg} / \mathrm{ml}$, Sigma Aldrich Co.) were added to each well. After $4 \mathrm{~h}$ incubation at $37^{\circ} \mathrm{C}$ in a $5 \% \quad \mathrm{CO}_{2}$ air atmosphere, the MTT solution was removed and the precipitated formazan was dissolved in dimethyl sulfoxide (DMSO). After several minutes of vacillation at room temperature, the absorbance of the formazan solution from the wells was calculated on an enzyme-linked immunosorbent assay (ELISA) reader at a wavelength of $570 \mathrm{~nm}$ using a microtiter plate reader (Anthos 2020 Microplate Readers, Biochrom Ltd., UK) and optical densities (ODs) were reported. A 620-nm filter was used for background reading.

\subsection{BMMSCs Proliferation Rate Assay}

MTT assay was performed to evaluate the proliferation of passage-4 sham BMMSCs (cells) and OVX cells separately incubated in a basic medium. In each well, 1000, 2000, 3000, 4000, 5000, 6000, and 7000 cells were seeded and the experiment was repeated five times. After incubation, the MTT solution was removed and the precipitated formazan was dissolved in DMSO. After oscillation, the absorbance of the formazan solution from the wells was computed on an ELISA reader at a wavelength of $570 \mathrm{~nm}$ using a microtiter plate reader. A simple regression analysis was used to model the relationship between the mean of ODs and the number of the cells in order to make a standard curve. For the study of the BMMSCs proliferation rate during the experiment, the mean of ODs was compared with their corresponding standard curves separately. Regarding significant results of the viability test, for the sham group, ODs of IR laser with energy density of $0.6 \mathrm{~J} / \mathrm{cm}^{2}$, and for the OVX group, the He-Ne laser with energy density of $1.2 \mathrm{~J} / \mathrm{cm}^{2}$ in days $2,4,6,14$, and 21 , were evaluated.

\subsection{Statistical Analysis}

After applying the Kolmogorov-Smirnov test to check the normality, we used a parametric approach to analyze our data. HU and ODs of OVX rats and sham rats were compared by Student $t$-test. Instead of using analysis of variance to compare the interesting factor effects, we would like to use $t$-test analysis to compare the treatments and their corresponding control group at each subgroup containing all combinations of the factor levels because our experiment contains a separate control group. We used confidence interval (CI) estimation for our purposes. Data are presented as mean \pm standard error of mean (SEM). A $p$ value equal to or less than 0.05 was considered as a statistically significant difference. We analyzed our data with SPSS 21.

\section{Results}

\subsection{Hounsfield Units Analysis}

Student $t$-test analysis revealed that there was a significant decrease in tibial bone density of OVX rats $(537 \pm 41.2 \mathrm{HU})$ as compared with the sham group $(679 \pm 25.3 \mathrm{HU})(p=0.000)$ and femoral bone density of OVX rats $(635.8 \pm 25.6 \mathrm{HU})$ as compared with the sham group $(787.8 \pm 24.4 \mathrm{HU})(p=0.000)$.

\subsection{Immunophenotyping on BMMSCs}

BMMSCs of the two groups were examined with an inverted phase contrast microscope (Nikon, Japan). Passage-4 BMMSCs had fibroblastic appearances with a homogenous spindle shape. Flow cytometry was used to determine the phenotype of BMMSCs, and cell surface markers of BMMSCs from passage- 4 were analyzed. The results showed that the cells of the two groups expressed at least $80 \%$ for the positive surface markers of BMMSCs (Fig. 3). The cells of OVX group expressed $64.9 \%$ for CD105. There was about $8 \%$ expression for negative markers of hematopoietic SCs.

\subsection{Effects of LLLT on Viability of Sham BMMSCs and OVX-BMMSCs}

\subsubsection{He-Ne laser}

Student $t$-test showed that there were significant differences in ODs of $1.2 \mathrm{~J} / \mathrm{cm}^{2}$ laser-treated sham group at day 21 and laser-treated OVX group with $0.6 \mathrm{~J} / \mathrm{cm}^{2}$ at day 2 as compared with their control groups ( $p=0.02$ and 0.01 , respectively) (Figs. 4 and 5).

\subsubsection{IR laser}

Student $t$-test showed that there were significant differences in ODs of $0.6 \mathrm{~J} / \mathrm{cm}^{2}$ laser-treated sham group at day 6 and 


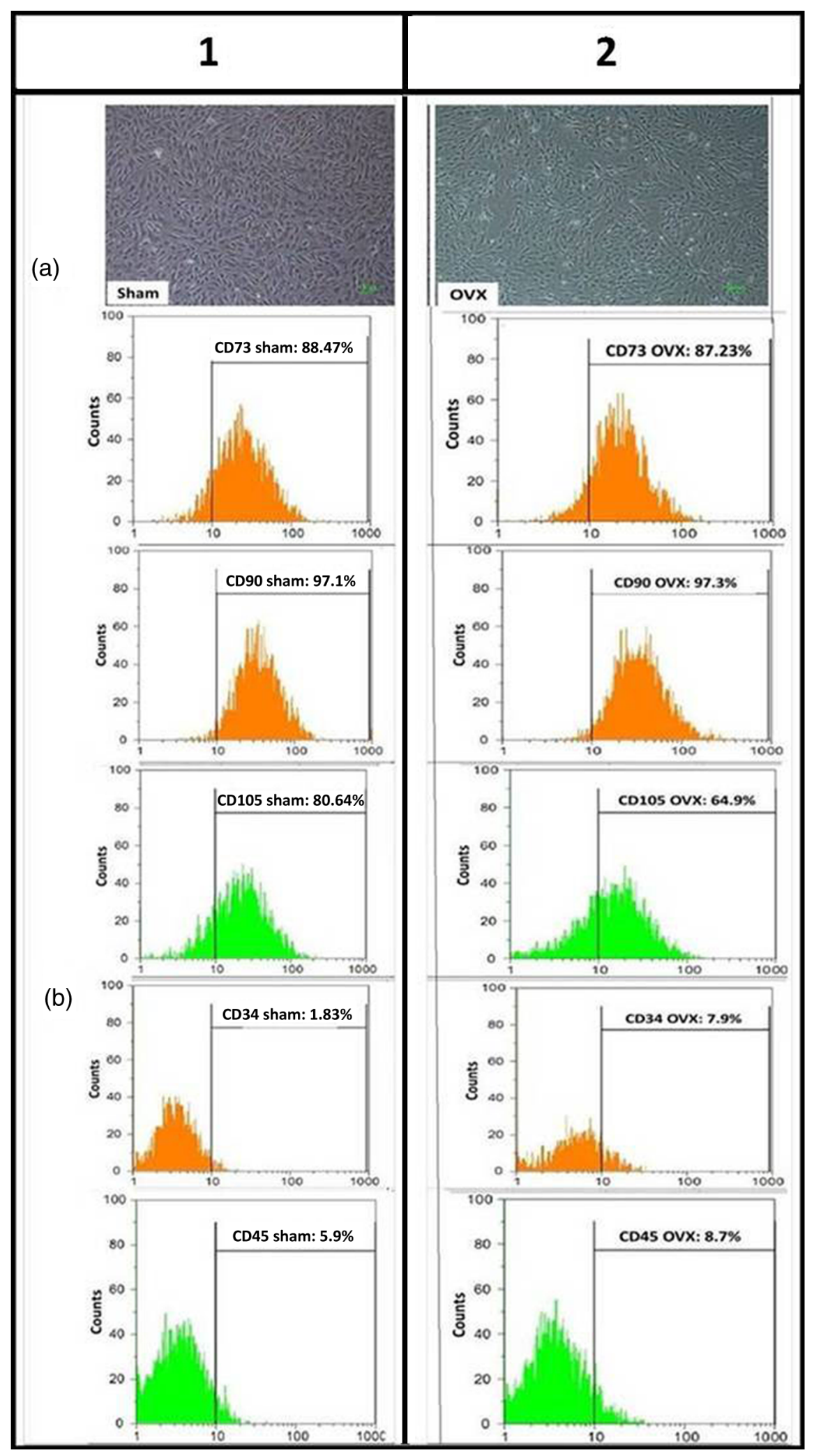

Fig. 3 Characterization of cultured BMMSCs. (a) Passage-4 BMMSCs grew as a spindle-shaped, fibroblastic, homogeneous monolayer cell population that had a characteristic, nonhematopoietic phenotype. (b) Immunophenotypes of the cells showed that they were positive for mesenchymal markers: CD105, CD90, and CD73, but did not express hematopoietic markers: CD34 and CD45. 


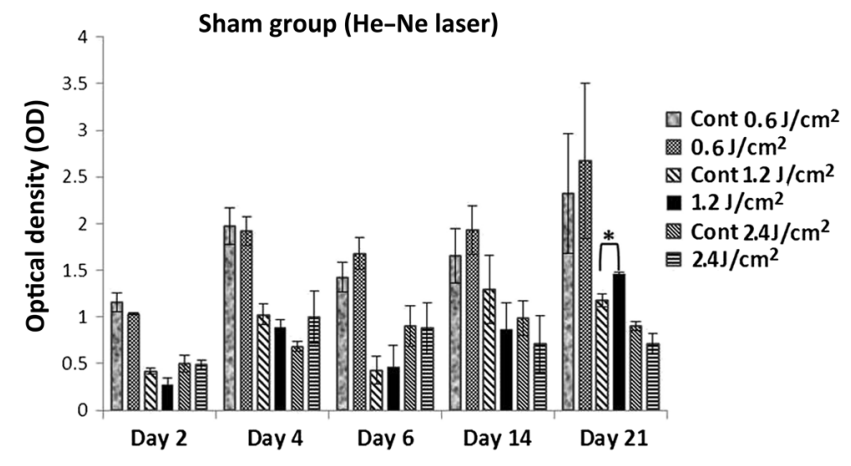

Fig. 4 Mean \pm SEM of ODs of helium-neon ( $\mathrm{He}-\mathrm{Ne}$ ) laser-treated sham BMMSCs; * $p<0.05$.

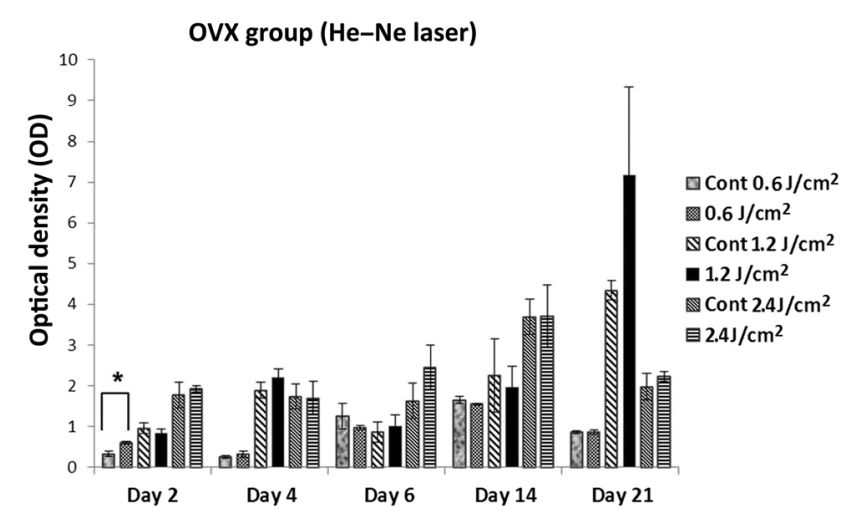

Fig. 5 Mean \pm SEM of ODs of He-Ne laser-treated ovariectomizedinduced OVX-BMMSCs; ${ }^{*} p<0.05$.

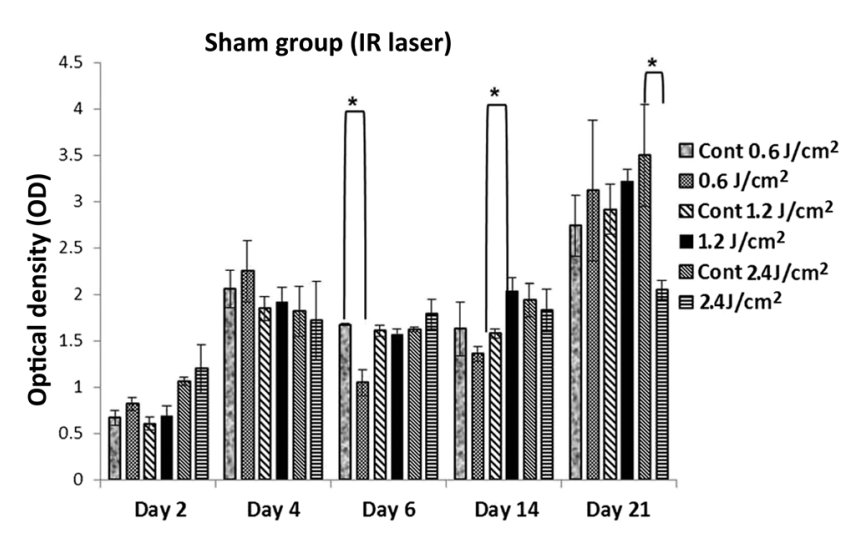

Fig. 6 Mean \pm SEM of ODs of IR laser-treated sham BMMSCs; ${ }^{*} p<0.05$.

$1.2 \mathrm{~J} / \mathrm{cm}^{2}$ at day 14 as compared with their control groups ( $p=0.01$ and 0.04, respectively) (Figs. 6 and 7).

\subsection{BMMSCs Proliferation Rate Analysis}

Figure 8 shows that the number of $\mathrm{He}-\mathrm{Ne}$ laser-treated BMMSCs in the OVX group started to increase significantly after day 6 in comparison with the IR laser-treated sham group. This result shows the comparison of the corresponding 95\% IR laser-treated sham's CI, 28.93 (23.37, 34.49; 95\% CI), and He-Ne laser-treated OVX's CI, 69.03 (49.51, 88.52; 95\% $\mathrm{CI}$ ), on day 21 , where the two intervals did not overlap.

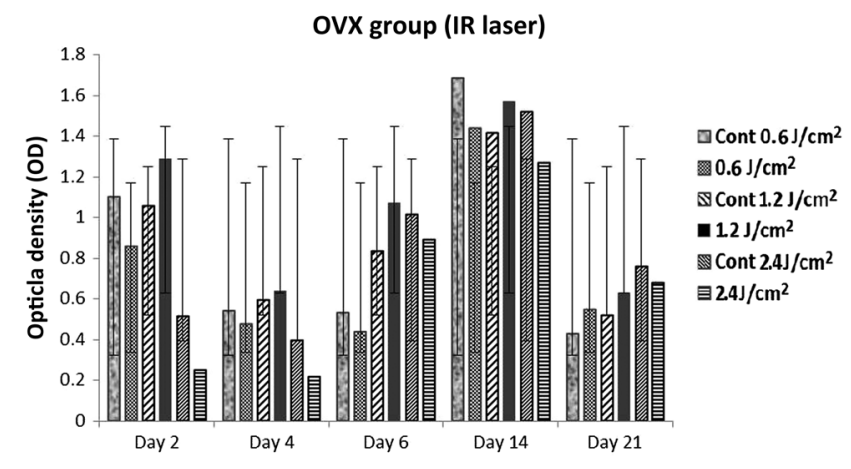

Fig. 7 Mean \pm SEM of ODs of IR laser-treated ovariectomizedinduced osteoporotic BMMSCs.

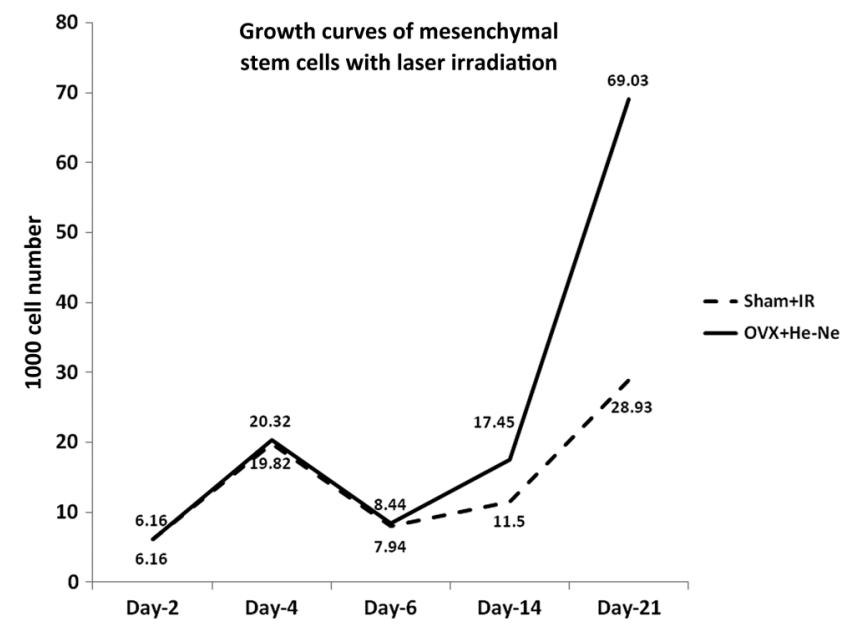

Fig. 8 The trends of the number of BMMSCs, separately, for the treatments: sham + IR laser (dotted line) and OVX + He-Ne laser (solid line) over the time (day factor). Although it seems the trends are not statistically different before day 6 , the number of $\mathrm{He}-\mathrm{Ne}$ laser-treated BMMSCs in the OVX + He-Ne laser group started to raise, significantly, after day 6 . This result comes from the corresponding $95 \%$ Cls of sham + IR laser, $28.93(23.37,34.49 ; 95 \% \mathrm{Cl})$, and OVX + $\mathrm{HE}-\mathrm{Ne}, 69.03(49.51,88.52 ; 95 \% \mathrm{Cl})$, on day 21 , where the two intervals have no overlap. Sham $+\mathrm{IR}=($ Sham $+\mathrm{IR}$ laser $), \mathrm{OVX}+\mathrm{He}-\mathrm{Ne}$ (OVX + He-Ne laser).

\section{Discussion}

In the present study, two types of LLLs' effects on viability and proliferation rate of BMMSCs extracted from healthy and osteoporotic rats were evaluated, respectively. The results showed that healthy BMMSCs responded optimally to $0.6 \mathrm{~J} / \mathrm{cm}^{2}$ using the IR laser. Moreover, it was found that OVX-BMMSCs responded optimally to $0.6 \mathrm{~J} / \mathrm{cm}^{2}$ with the $\mathrm{He}-\mathrm{Ne}$ laser.

Estrogen plays an important role in the maintenance of stable bone mass during adult life. ${ }^{28}$ In the current study, the authors used sham and OVX rats, which are most commonly used as experimental and control animal models for postmenopausal $\mathrm{OP}^{29}$ and the duration from bilateral ovariectomization to the extraction, isolation, and culture of BMMSCs was 3.5 months. ${ }^{24,25}$ This model has also been approved by the Food and Drug Administration to be the primary model system for preclinical evaluation of agents used for the prevention and treatment of postmenopausal OP. ${ }^{30}$ BMMSC from postmenopausal women with OP showed significant decreases in cell growth, proliferative response, and osteogenic differentiation. ${ }^{31}$ In a study on OVX rats, BMMSCs isolated from osteoporotic rats showed 
less differentiation capabilities as compared with those isolated from healthy rats. The results clearly demonstrated the importance of BMMSCs in OP and that the disease can be treated by injection with BMMSCs. ${ }^{32}$ On the other hand, autologous MSCs expanded in vitro are the potential strategy to augment bone formation in elderly subjects suffering from OP. The harvest of a limited BM sample (of a healthy person) is an easy and relatively safe procedure. Large numbers of BMMSCs can be obtained in culture, making it possible to cause transplantable constructs composed of these cells in appropriate scaffolds. ${ }^{33}$ As a primitive cell population, BMMSCs have the potential to produce bone tissue. Therefore, the regenerative potential of BMMSCs is highly dependent on the cellular and molecular mechanisms that underlie the lineage differentiation of BMMSCs and their responses to exogenous stimulation. ${ }^{34}$ On the other hand, the issue of in vitro preconditioning of BMMSCs and especially OVX-BMMSCs former transplantation to improve these methods for MSC-based regeneration is a fascinating approach in the field of regenerative medicine.

Our results showed that an IR laser can be employed in the stimulation of healthy BMMSCs viability in vitro and to improve their regenerative potentials, producing the cells to be transplanted in the right quantity in the injured tissue. ${ }^{18}$ These results are in agreement with previous studies. ${ }^{20,21}$ LLLT $\left(810 \mathrm{~nm}, 2\right.$ to $6 \mathrm{~J} / \mathrm{cm}^{2}, 50 \mathrm{~mW}, \sim 167 \mathrm{~mW} / \mathrm{cm}^{2}$ ) not only enhanced BMMSCs differentiation into neurons and osteoblasts, but also increased BMMSCs' proliferation (except for $\left.6 \mathrm{~J} / \mathrm{cm}^{2}\right) .{ }^{20}$ It is concluded that the effect of LLLT on differentiation and proliferation of BMMSCs is dose-dependent. ${ }^{20}$ The effect of LLLT (Ga-Al-Ar, diode laser, $808 \mathrm{~nm}, 50 \mathrm{~mW}$, three times a week) on mice BM cells proliferation was studied. ${ }^{21}$ Bouvet-Gerbettaz et al. ${ }^{21}$ found that LLL did not alter mice bone progenitor cell proliferation and their results could be related to the wavelength selected $(808 \mathrm{~nm})$, the energy density $\left(4 \mathrm{~J} / \mathrm{cm}^{2}\right)$, or the cell culture model, which has never been used before. They suggested that the laser biostimulation efficiency depends on the delivered energy density, which seems to be restricted to a very narrow therapeutic window. Similarly, in the current work, there were no significant differences between IR laser-treated healthy BMMSCs with $2.4 \mathrm{~J} / \mathrm{cm}^{2}$ and the control group. The results of the present study showed that healthy BMMSCs exposed to 0.6 and $1.2 \mathrm{~J} / \mathrm{cm}^{2}$ with $810 \mathrm{~nm}$ for three times showed a statistically significant increase in viability when compared with osteoporotic-BMMSCs exposed to 0.6 to $2.4 \mathrm{~J} / \mathrm{cm}^{2}$ with $810 \mathrm{~nm}$ for three times. Based on our findings, a $\mathrm{He}-\mathrm{Ne}$ laser can be employed in the stimulation of osteoporotic BMMSCs' viability and proliferation in vitro and to improve their regenerative potentials, producing the cells to be transplanted in the proper quantity and quality in the injured tissue. A He-Ne laser with $1.2 \mathrm{~J} / \mathrm{cm}^{2}$ can be employed to stimulate healthy BMMSCs' viability and proliferation in vitro. However, its effect was statistically lower than that of the IR laser (He-Ne laser: $p=0.02$ versus IR laser: $p=0.01$ ). Recently, stimulation with a He-Ne laser $\left(2 \mathrm{~J} / \mathrm{cm}^{2}\right)$ caused a significant increase in human epidermal SCs' migration and proliferation in vitro, which probably contributed, at least partly, to accelerated wound re-epithelialization with a low-level laser. ${ }^{18}$ The cellular mechanisms associated with the biostimulatory effects of the He-Ne laser on SCs have not been elucidated. In other cells, one of the possible reasons may be that the laser energy is absorbed by intracellular chromophores and converted to metabolic energy. Cellular ATP levels increased by almost $190 \%$ after $\mathrm{He}-\mathrm{Ne}$ laser radiation $\left(100 \mathrm{~mJ}^{-2}, 8 \mathrm{~s}\right)$ in cultured HeLa cells. ${ }^{35}$ Moreover, the ability of monochromatic red light to induce an increase in the cellular ATP level was found to depend on the growth phase of the cultured cells, being insignificant in the lag phase of cultured cells, increasing in the log phase of cultured cells, and reaching a maximum (about 190\%) in cells at the late logarithmic and early plateau phases. ${ }^{34}$

In the current study, osteoporotic BMMSCs exposed to $0.6 \mathrm{~J} / \mathrm{cm}^{2}$ using an $\mathrm{He}-\mathrm{Ne}$ laser for three times showed a statistically significant increase in the viability when compared with the healthy BMMSCs exposed to $1.2 \mathrm{~J} / \mathrm{cm}^{2}$ with a $\mathrm{He}-\mathrm{Ne}$ laser for three times.

LL red light (range 630 to $690 \mathrm{~nm}$ ) as generated by LLLs or light-emitting diode (LED) arrays has been reported to stimulate MSC. ${ }^{35,36}$

The effect of light from a red LED upon osteogenic differentiation of mouse MSCs (D1 cells) cultured in the presence of osteogenic differentiation medium (ODM), then exposed to a red LED light with $647 \mathrm{~nm}$ once for 10,30 , or $90 \mathrm{~s}$, with radiation energies of $0.093,0.279$, and $0.836 \mathrm{~J}$, respectively, was reported. ${ }^{36} \mathrm{D} 1$ cells differentiated into osteoblasts in the presence of ODM, and this process was increased on exposure to LED light in an ODM medium. The authors concluded that osteogenic differentiation of MSCs in ODM is enhanced by LED light exposure. But the real benefits of LED light must be established by further investigations using well-controlled protocols. ${ }^{36}$ The growth of rat bone MSCs that were treated by single or multiple doses of LLL from an LED array $\left(630 \mathrm{~nm}, 2\right.$ and $4 \mathrm{~J} / \mathrm{cm}^{2}, 5$ and $\left.15 \mathrm{~mW} / \mathrm{cm}^{2}\right)$ was enhanced by red light LLL and the effect became more obvious at low cell density. Furthermore, a single dose of LLLT only led to a short-term acceleration in the MSCs' proliferation, but a maximum acceleration in cell proliferation was observed during multiple exposures of LLLT at $15 \mathrm{~mW} / \mathrm{cm}^{2}$ and $4 \mathrm{~J} / \mathrm{cm}^{2}{ }^{36,37}$ The Hawkins and Abrahamse ${ }^{38}$ study suggests that the duration of the laser effect should be considered when interpreting and reporting the cellular responses such as viability and proliferation. These in vitro results may be useful in understanding different biostimulatory or bioinhibitory reactions prevailing in cases of lower and higher doses at different wavelengths. In the current study, with the application of a He-Ne laser for three times on healthy BMMSCs of the sham group, the cells exhibited a significant higher viability within 21 days after radiation when compared with the nonirradiated cells. According to the results of a single exposure of the He-Ne laser to OVXBMMSCs of OVX group, significant cell viability increased at 2 days after radiation as compared with the control group. It seems that the physiologic state of BMMSCs (healthy or osteoporotic) and energy density of the He-Ne laser play an important role in determining the long-term effects of the laser on BMMSCs in vitro.

The use of SCs has shown effective results in the treatment of conditions that vary from cartilage defects in joints to hematological malignancy. In OP, some of the treatment options include bone regeneration with growth factors such as recombinant human BMPs or genetic alteration of cells to modify phenotypes. ${ }^{39}$ Despite the fact that gene therapy may be one of the methods available to orthopedic surgeons in treating challenging bone problems in $\mathrm{OP}$, the safety issues concerning gene therapy are still indefinite, whereas the implantation of autologous cells is becoming useful for clinical practice. ${ }^{22}$ The results of this study indicated that LLLT significantly increased the 
viability and proliferation rate of OVX-BMMSCs. Thus, further investigations on transplanting in vitro cultured improved autologous BMMSCs into sites particularly at risk of developing osteoporotic bone are suggested. This technique may improve bone quality and structure.

Recently, Li et al. established a standard model of OVX in rats. Female adult rats were bilateral ovariectomized and left untreated for 3.5 months, allowing for the development of OP. Using a CT system, volumetric bone mineral content (vBMC), vBMD, and microarchitecture of the fifth lumbar vertebra and femur-tibia sites were examined. Mechanical properties of the fifth lumbar vertebra and femur-tibia sites were measured. The results confirmed OP at both the lumbar vertebrae and femurtibia sites in OVX rats compared with age-matched sham controls. This rat experimental model of OP was established to be a model for individuals with estrogen deficiency-induced OP. Other related studies confirm the Li et al. model of OP. Therefore, in the current study, we used this experimental animal model to induce an established model of OP in rats. ${ }^{25}$

There is increasing evidence in the literature that information about bone quality can also be determined via HU measurements obtained from diagnostic CT scans. ${ }^{40,41}$ An HU value is a standardized linear attenuation coefficient of tissue, based on a defined scale of 0 for water, -1000 for air, and +1000 for bone, that represents the density of tissue. HU measurements can be calculated from a region of interest using most modern radiology imaging software without added costs or radiation. Measuring HU from CT has generated authentic estimates of BMD in the spine. ${ }^{42}$

Assessing local bone quality on CT scans with HU quantification is being used with increasing frequency. Correlations between HU and BMD have been determined, and normative data have been illustrated throughout the spine. The information provided by a simple HU measurement can not only alert the treating physician to decreased bone quality, but can also be useful in both medically and surgically managing these patients. ${ }^{43}$

Many studies have used HU for comparing data between control and experimental groups in animal models. James et al. $^{44}$ demonstrate that NELL-1-haploinsufficient mice have normal development in their skeleton, but go through agerelated OP. They induced OP in male mice by gonadectomy and provided systemic administration of NELL-1 to experimental mice. They found improvement in BMD, which is measured by HU. ${ }^{44}$ Shakouri et al. evaluated the effect of LLLT on fracture healing in rabbits. The animals were divided into laser and control groups. Callus development and BMD were quantitatively evaluated by CT scanning using a multidetector (64MD) Siemens scanner. Data were reported in HU. The results demonstrated that the increasing rate of BMD was higher in the laser group than in the control group. CT revealed a mean callus density of $297 \pm 337 \mathrm{HU}$ for the control group and $691 \pm 143 \mathrm{HU}$ for the laser group, which was statistically significant. ${ }^{26}$ Cankaya et al. investigated the clinical and histopathological presentation of bisphosphonate-induced osteonecrosis of the jaw with the HU and assessed the reliability of the HU for ascertaining necrosis in the animal model. They divided rats into control and experimental groups. Half of the animals from each group went through extraction of the left mandibular molars, and the other half underwent extraction of the left maxillary molars. After 28 days, maxillae and mandibles were extracted. Cone beam CT (CBCT) was performed and HU was evaluated. Cankaya et al. found that the HU for maxillae and mandibles of the experimental groups $(249.2 \pm 80.2,435.4 \pm 61.1)$ were significantly lower than that of the control groups $(827.1 \pm 20.3$, $868.3 \pm 49.2){ }^{45}$ Therefore, in the current study, we first induced bilateral ovairectomy in rats. Second, ovariectomized rats were left untreated for 3.5 months, allowing for the development of OP. Third, OP development of OVX rats was evaluated qualitatively by CT scanning using multislides (Toshiba, Japan) as compared with the aged matched sham rats. Finally, bone densities were reported in HU. Our statistical analysis revealed that there was a significant decrease in bone density of OVX rats as compared with the sham group.

In order to get a reference value of the BMD of patients, researchers not only perform special studies for each case, but also report a reference value for that case. For example, Pickhardt et al. evaluated CT-derived BMD assessment compared with dual-energy x-ray absorptiometry (DXA) measures for identifying OP using CT scans performed for other clinical indications. One thousand eight hundred and sixty seven adults undergoing CT scanning and DXA ( $n=2067$ pairs) within a 180-day period over 10 years participated in the study. CT-attenuation values (in $\mathrm{HU}$ ) of trabecular bone between the T12 and L5 vertebral levels were measured by DXA-BMD as the reference standard. Sagittal CT scanning images assessed for moderate-to-severe vertebral fractures. Pickhardt et al. demonstrated that CT-attenuation values were significantly lower at all vertebral levels for patients with DXA-defined OP. An L1 CT-attenuation threshold of $160 \mathrm{HU}$ or less was $90 \%$ sensitive and a threshold of $110 \mathrm{HU}$ was more than $90 \%$ specific for determining OP from osteopenia and normal BMD. Positive predictive values for OP were $68 \%$ or greater at L1 CT-attenuation thresholds less than $100 \mathrm{HU}$; negative predictive values were $99 \%$ at thresholds greater than $200 \mathrm{HU}$. Pickhardt et al. concluded that abdominal CT images obtained for other reasons that include the lumbar spine can be used to identify patients with OP or normal BMD without additional radiation exposure or cost. ${ }^{40}$ We concluded that these measurements and their reference values for $\mathrm{HU}$ were more suitable for patients. On the other hand, it seems that in animal studies comparing HU of experimental groups with their matched control groups gives valid and reliable results.

\section{Conclusion}

LLLT can enhance the viability and proliferation rate of healthy and especially osteoporotic autologous BMMSCs that could be very useful in regenerative medicine for the treatment of OP and fracture of patients who are OP-dependent. LLLT effect depends on the physiological state of the BMMSCs, type of the laser, radiation wavelength, energy density, and number of laser sessions. The biostimulation efficiency of LLLT also depends on the delivered energy density, which seems to be restricted to a very narrow therapeutic window. The results of this study showed that healthy BMMSCs responded optimally to an IR laser with $6 \mathrm{~J} / \mathrm{cm}^{2}$ after three times laser radiation. Moreover, it was found that OVX-BMMSCs responded optimally to $0.6 \mathrm{~J} / \mathrm{cm}^{2}$ with an $\mathrm{He}-\mathrm{Ne}$ laser and one time laser radiation. We suggest using other wavelengths and energy densities of LLLT and other techniques for assaying cellular proliferation such as a DNA-based proliferation assay in further studies. On the other hand, it was concluded that animal studies comparing the HU of experimental groups with their matched control groups gives valid and reliable results. 


\section{Acknowledgments}

The authors wish to extend their sincere thanks to late Mrs. Jamileh Rezaei. This article was financially supported by the Research Department of the Shahid Beheshti University of Medical Sciences, Tehran, Iran (Grant No. 1393-1-91-13237).

\section{References}

1. J. P. Bidwell et al., "Functional impairment of bone formation in the pathogenesis of osteoporosis: the bone marrow regenerative competence," Curr. Osteoporosis Rep. 11(2), 117-125 (2013).

2. A. L. Golob and M. B. Laya, "Osteoporosis: screening, prevention, and management," Med. Clin. North Am. 99(3), 587-606 (2015).

3. A. M. Pino, C. J. Rosen, and J. P. Rodríguez, "In osteoporosis, differentiation of mesenchymal stem cells (MSCs) improves bone marrow adipogenesis," Biol. Res. 45(3), 279-287 (2012).

4. K. P. Chang et al., "Incidence of hip and other osteoporotic fractures in elderly men and women: Dubbo Osteoporosis Epidemiology Study," J. Bone Miner. Res. 19(4), 532-536 (2004).

5. D. J. Becker, M. L. Kilgore, and M. A. Morrisey, "The societal burden of osteoporosis," Curr. Rheumatol. Rep. 12(3), 186-191 (2010).

6. W. C. Prall et al., "Mesenchymal stem cells from osteoporotic patients feature impaired signal transduction but sustained osteoinduction in response to BMP-2 stimulation," Biochem. Biophys. Res. Commun. 440(4), 617-622 (2013).

7. F. Haasters et al., "Mesenchymal stem cells from osteoporotic patients reveal reduced migration and invasion upon stimulation with BMP-2 or BMP-7," Biochem. Biophys. Res. Commun. 452(1), 118-123 (2014).

8. D. Tewari et al., "Ovariectomized rats with established osteopenia have diminished mesenchymal stem cells in the bone marrow and impaired homing, osteoinduction and bone regeneration at the fracture site," Stem Cell Rev. Rep. 11(2), 309-321 (2015).

9. F. Ginani, D. M. Soares, and C. A. G. Barboza, "Effect of low-level laser therapy on mesenchymal stem cell proliferation: a systematic review," Lasers Med. Sci. 30(8), 2189-2194 (2015).

10. Y.-Y. Huang et al., "Biphasic dose response in low level light therapy," Dose-Response 7(4), 358-383 (2009).

11. T. Karu, "Laser biostimulation: a photobiological phenomenon," J. Photochem. Photobiol., B 3(4), 638 (1989).

12. T. Karu, "Primary and secondary mechanisms of action of visible to near-IR radiation on cells," J. Photochem. Photobiol., B 49(1), 1-17 (1999).

13. G. K. Reddy, "Photobiological basis and clinical role of low-intensity lasers in biology and medicine," J. Clin. Laser Med. Surg. 22(2), 141150 (2004).

14. A. N. Silva, Jr. et al., "Computerized morphometric assessment of the effect of low-level laser therapy on bone repair: an experimental animal study," J. Clin. Laser Med. Surg. 20(2), 83-87 (2002).

15. A. C. M. Renno et al., "The effects of infrared- $830 \mathrm{~nm}$ laser on exercised osteopenic rats," Lasers Med. Sci. 21(4), 202-207 (2006).

16. A. Renno et al., "The effects of laser irradiation on osteoblast and osteosarcoma cell proliferation and differentiation in vitro," Photomed. Laser Surg. 25(4), 275-280 (2007).

17. F. P. Barry and J. M. Murphy, "Mesenchymal stem cells: clinical applications and biological characterization," Int. J. Biochem. Cell Biol. 36(4), 568-584 (2004).

18. X. Liao et al., "Helium-neon laser irradiation promotes the proliferation and migration of human epidermal stem cells in vitro: proposed mechanism for enhanced wound re-epithelialization," Photomed. Laser Surg. 32(4), 219-225 (2014).

19. H. Tuby, L. Maltz, and U. Oron, "Low-level laser irradiation (LLLI) promotes proliferation of mesenchymal and cardiac stem cells in culture," Lasers Surg. Med. 39(4), 373-378 (2007).

20. M. Soleimani et al., "The effects of low-level laser irradiation on differentiation and proliferation of human bone marrow mesenchymal stem cells into neurons and osteoblasts—an in vitro study," Lasers Med. Sci. 27(2), 423-430 (2012).

21. S. Bouvet-Gerbettaz et al., "Effects of low-level laser therapy on proliferation and differentiation of murine bone marrow cells into osteoblasts and osteoclasts," Lasers Surg. Med. 41(4), 291-297 (2009).
22. Z. Wang et al., "Efficacy of bone marrow-derived stem cells in strengthening osteoporotic bone in a rabbit model," Tissue Eng. 12(7), 17531761 (2006).

23. M. D. Griffin, T. Ritter, and B. P. Mahon, "Immunological aspects of allogeneic mesenchymal stem cell therapies," Hum. Gene Ther. 21(12), 1641-1655 (2010).

24. M. Fridoni et al., "Evaluation of the effects of LLLT on biomechanical properties of tibial diaphysis in two rat models of experimental osteoporosis by a three point bending test," Lasers Med. Sci. 30(3), 1117-1125 (2015).

25. X. Li et al., "Increased bone formation and bone mass induced by sclerostin antibody is not affected by pretreatment or cotreatment with alendronate in osteopenic, ovariectomized rats," Endocrinology 152(9), 3312-3322 (2011).

26. S. K. Shakouri et al., "Effect of low-level laser therapy on the fracture healing process," Lasers Med. Sci. 25(1), 73-77 (2010).

27. M. Esmaeelinejad et al., "The effects of low-level laser irradiation on cellular viability and proliferation of human skin fibroblasts cultured in high glucose mediums," Lasers Med. Sci. 29(1), 121-129 (2014).

28. X. Jiang and P. F. Schnatz, "Menopausal medicine: how to educate the next generation of menopause clinicians," Menopause 20(11), 1109-1110 (2013)

29. A. S. Turner, "Animal models of osteoporosis-necessity and limitations," Eur. Cell Mater. 1, 66-81 (2001).

30. E. G. Colman, "The Food and Drug Administration's osteoporosis guidance document: past, present, and future," J. Bone Miner. Res. 18(6), 1125-1128 (2003).

31. J. P. Rodríguez et al., "Abnormal osteogenesis in osteoporotic patients is reflected by altered mesenchymal stem cells dynamics," J. Cell. Biochem. 75(3), 414-423 (1999).

32. NdM. Ocarino et al., "Intra-bone marrow injection of mesenchymal stem cells improves the femur bone mass of osteoporotic female rats," Connect. Tissue Res. 51(6), 426-433 (2010).

33. R. Cancedda et al., "Tissue engineering and cell therapy of cartilage and bone," Matrix Biol. 22(1), 81-91 (2003).

34. Q. Shen et al., "Curculigoside promotes osteogenic differentiation of bone marrow stromal cells from ovariectomized rats," J. Pharm. Pharmacol. 65(7), 1005-1013 (2013).

35. T. Karu, L. Pyatibrat, and G. Kalendo, "Irradiation with $\mathrm{He}-\mathrm{Ne}$ laser increases ATP level in cells cultivated in vitro," J. Photochem. Photobiol., B 27(3), 219-223 (1995).

36. W.-T. Li, Y.-C. Leu, and J.-L. Wu, "Red-light light-emitting diode irradiation increases the proliferation and osteogenic differentiation of rat bone marrow mesenchymal stem cells," Photomed. Laser Surg. 28(S1), S-157-S-165 (2010).

37. H. K. Kim et al., "Red light of $647 \mathrm{~nm}$ enhances osteogenic differentiation in mesenchymal stem cells," Lasers Med. Sci. 24(2), 214-222 (2009).

38. D. H. Hawkins and H. Abrahamse, "Time-dependent responses of wounded human skin fibroblasts following phototherapy," J. Photochem. Photobiol., B 88(2), 147-155 (2007).

39. K. Nakamura et al., "Stimulation of bone formation by intraosseous application of recombinant basic fibroblast growth factor in normal and ovariectomized rabbits," J. Orthop. Res. 15(2), 307-313 (1997).

40. P. J. Pickhardt et al., "Opportunistic screening for osteoporosis using abdominal computed tomography scans obtained for other indications," Ann. Intern. Med. 158(8), 588-595 (2013).

41. O. Emohare et al., "Osteoporosis in acute fractures of the cervical spine: the role of opportunistic CT screening," J. Neurosurg. 23(1), 1-7 (2015).

42. C. C. Johnson et al., "Using Hounsfield units to assess osteoporotic status on wrist computed tomography scans: comparison with dual energy x-ray absorptiometry," J. Hand Surg. 41(7), 767-774 (2016).

43. J. J. Schreiber, P. A. Anderson, and W. K. Hsu, "Use of computed tomography for assessing bone mineral density," Neurosurg. Focus 37(1), E4 (2014).

44. A. W. James et al., "NELL-1 in the treatment of osteoporotic bone loss," Nat. Commun. 6, 7362 (2015).

45. A. B. Cankaya et al., "Use of cone-beam computerized tomography for evaluation of bisphosphonate-associated osteonecrosis of the jaws in an experimental rat model," Int. J. Med. Sci. 8(8), 667 (2011).

Biographies for the authors are not available. 